\title{
Expression of the zinc finger transcription factor Sp6-9 in the velvet worm Euperipatoides kanangrensis suggests a conserved role in appendage development in Panarthropoda
}

\author{
Ralf Janssen $^{1}$ (D) $\cdot$ Graham E. Budd ${ }^{1}$ \\ Received: 29 March 2020 / Accepted: 11 May 2020 / Published online: 19 May 2020 \\ (C) The Author(s) 2020
}

\begin{abstract}
The Sp-family genes encode important transcription factors in animal development. Here we investigate the embryonic expression patterns of the complete set of Sp-genes in the velvet worm Euperipatoides kanangrensis (Onychophora), with a special focus on the Sp6-9 ortholog. In arthropods, Sp6-9, the ortholog of the Drosophila melanogaster D-Sp1 gene plays a conserved role in appendage development. Our data show that the expression of Sp6-9 during the development of the velvet worm is conserved, suggesting that the key function of the Sp6-9 gene dates back to at least the last common ancestor of arthropods and onychophorans and thus likely the last common ancestor of Panarthropoda.
\end{abstract}

Keywords Arthropod development $\cdot$ Appendage development $\cdot$ Sp1 $\cdot$ SP5 $\cdot$ Buttonhead $\cdot$ Panarthropoda $\cdot$ Onychophora

\section{Introduction}

Sp-family genes encode a conserved group of transcription factors, all of which possess three highly conserved $\mathrm{C}_{2} \mathrm{H}_{2}$-type zinc fingers that bind to G-rich regions in their target genes and a so-called buttonhead box (reviewed in, e.g. Kadonaga et al. 1987; Suske 1999; Kolell and Crawford 2002; Schaeper et al. 2010). The name "Sp" originates from the technique used to first purify an "SP" gene using Sephacryl columns and phosphocellulose chromatography (Dynan and Tjian 1983). It has been shown that Sp genes bind to a plethora of target genes and are thus involved in many developmental processes (reviewed in, e.g. Suske et al. 2005). The last common ancestor of all metazoans already possessed three $\mathrm{Sp}$ genes (Schaeper et al. 2010). In vertebrates, however, gene

Communicated by Nico Posnien

Electronic supplementary material The online version of this article (https://doi.org/10.1007/s00427-020-00661-w) contains supplementary material, which is available to authorized users.

Ralf Janssen

ralf.janssen@geo.uu.se

1 Department of Earth Sciences, Uppsala University, Palaeobiology, Villavägen 16, Uppsala, Sweden duplication has led to a much larger complement of Sp genes, and since much of the initial research on Sp genes comes from vertebrates, the nomenclature of Sp genes may to some degree be confusing. Sp genes are named $\mathrm{Sp} 1$ to $\mathrm{Sp} 9$. Sp1 to Sp4 are related and form the first clade (Sp1-4), Sp5 is the only member of its own clade, and Sp6 to Sp9 form the third clade (Sp69) (Suske et al. 2005; Zhao and Meng 2005; Schaeper et al. 2010; Suske 2017).

In arthropods, the situation is much simpler: there is one gene representing each class, Sp1-4, Sp5 and Sp6-9 (Schaeper et al. 2010). The first Sp gene to be identified and studied in the model arthropod Drosophila melanogaster was buttonhead (btd), which represents the mammalian Sp5 ortholog (Wimmer et al. 1993). The single arthropod member of the Sp1-Sp4 clade (Sp1-4) (CG5669 in Drosophila) is expressed ubiquitously and at equal levels during development, and it may thus represent a universal transcription factor (summarized in Schaeper et al. 2010). The third arthropod Sp gene is the single ortholog of the Sp6 to Sp9 clade (Sp6-9) (called D-Spl in Drosophila) (Wimmer et al. 1996). Drosophila btd (Sp5) and D-Sp1 (Sp6-9) are expressed in similar patterns in post-blastoderm stages, and it has been shown that the two genes can at least partially substitute for each another (Wimmer et al. 1996; Schöck et al. 1999). Subsequent research, however, has shown that it is $S p 6-9$ that represents a key factor of appendage development in 
Drosophila, a function that is not fully shared by Sp5/btd (Estella and Mann 2010; Cordoba et al. 2016). Functional studies in Drosophila (Cordoba et al. 2016) and other arthropods including sequentially segmenting insects such as the red flour beetle Tribolium castaneum (Beermann et al. 2004), the true bug Oncopeltus fasciatus (Schaeper et al. 2009) and the spider Parasteatoda tepidariorum (Königsmann et al. 2017; Setton and Sharma 2018) showed that limb growth is heavily disturbed in a $S p 6-9$ depleted or knock-down background, suggesting that the role of Sp6-9 as a key factor in limb growth is conserved among Arthropoda as a whole.

In this paper, we present the gene expression patterns of the three Sp genes, Sp1-4, Sp5/buttonhead-like (Sp5/btdl) and Sp6-9 in the onychophoran Euperipatoides kanangrensis. Onychophorans are closely related to arthropods and may indeed represent their sister group (e.g. Campbell et al. 2011), although the relationship of Onychophora, Tardigrada and Arthropoda is not fully resolved yet (reviewed in Giribet and Edgecombe 2017).

The data show that the role of $S p 6-9$ in appendage growth is likely conserved in onychophorans and thus likely in all of Panarthropoda. The expression of the onychophoran Sp5/btdl ortholog suggests a certain degree of redundancy of the more closely related Sp genes $S p 6-9$ and $S p 5 / b t d l$ (Schaeper et al. 2010), as reported for D-Sp1 (Sp6-9) and btd (Sp5) in Drosophila (Wimmer et al. 1996; Schöck et al. 1999). As in arthropods, the onychophoran $\mathrm{Sp} 5 / \mathrm{btdl}$ gene may play an early role during germ band patterning, albeit not, unlike in arthropods, restricted to head development. Unlike the situation in arthropods, the onychophoran Sp1-4 gene is not expressed ubiquitously but superficially resembles the patterns of $S p 5 / b t d$ and $S p 6-9$.

\section{Methods}

\section{Animal husbandry and fixation of embryos}

Embryos were obtained and treated for subsequent in situ hybridization experiments as described in Hogvall et al. (2014). Developmental stages are described in Janssen and Budd (2013).

\section{Phylogenetic analysis}

Sp-family genes were identified performing reciprocal BLAST searches against the sequenced embryonic transcriptome of Euperipatoides using the sequences of Drosophila sp. orthologs as baits.

Amino acid sequences of the conserved regions of putative Spgenes, and the Cabut protein sequence of Drosophila that serves as an outgroup sequence, were aligned using T-Coffee followed by manual editing in SeaView (Notredame et al. 2000; Gouy et al. 2010) using default parameters as suggested for MacVector v12.6.0 (MacVector, Inc., Cary, NC). A Bayesian phylogenetic analysis was executed in MrBayes (Huelsenbeck and Ronquist 2001) with a fixed WAG amino acid substitution model with gamma-distributed rate variation across sites (with four rate categories), unconstrained exponential prior probability distribution on branch lengths and exponential prior for the gamma shape parameters for among site rate variation. The tree topology was calculated applying 500,000 cycles for the Metropolis-Coupled Markov Chain Monte Carlo (MCMCMC) analysis (four chains; chainheating temperature of 0.2 ). Markov chains were sampled every 200 cycles. Default settings were used, defining $25 \%$ of the samples as burn-in information. Clade support was calculated with posterior probabilities in MrBayes. Unique sequence identifiers of all sequences used in the analysis are listed in Supplementary File F1. The nexus file and the alignment are available as Supplementary Files F2 and F3.

\section{Gene cloning, whole mount in situ hybridization and nuclear staining}

Sections of SP-genes were amplified by RT-PCR with genespecific primers that were based on the information from the sequenced embryonic transcriptome of Euperipatoides (PRJNA525753: SRR8690378). For all genes, nested PCRs were run with internal primers, using $1 \mu \mathrm{l}$ of the product of a first PCR as template. Primer sequences are listed in Supplementary File F4. All gene fragments were cloned into the PCRII vector (Invitrogen) and sequenced on an ABI3730XL automatic sequencer (Macrogen, Seoul, South Korea). Gene-identifying numbers are summarized in Supplementary File F1. In situ hybridizations were performed using a universally working protocol that is described in Janssen et al. (2018, supplement). Cell nuclei were stained with 1:10000 SYBR-Green (Invitrogen) in phosphate buffered saline with $0.1 \%$ Tween-20 (PBST-0.1\%) for approximately $20 \mathrm{~min}$ at room temperature.

\section{Data documentation}

Bright-field microscopy and visualization of SYBR-Green stain were performed with a Leica-DC490 digital camera that was equipped with a UV light source mounted onto a MZFLIII Leica dissection microscope. Linear adjustments of colour contrast and brightness were executed using the imageprocessing software Adobe Photoshop CS6 for Apple Macintosh (Adobe Systems Inc.)

\section{Results and discussion}

\section{Sequence analysis}

Three Sp-family genes were identified in Euperipatoides (Fig. 1) suggesting that onychophorans possess the full 
complement of Sp genes (Schaeper et al. 2010). As in other phylogenetic analyses (Schaeper et al. 2009, 2010; Königsmann et al. 2017), members of the Sp1-4 and Sp6-9 clade cluster with high confidence, while members of the more-derived buttonhead (Btd) and Sp5 clade often cluster with low support or remain unresolved, as in this study (Fig. 1). The onychophoran Sp5/btdl sequence, however, clusters with the previously described $b t d$-like (btdl) sequence from the myriapod Glomeris marginata (Janssen et al. 2011). In some arthropod species, especially chelicerates, $S p 5$ and $b t d$-like genes appear to have been frequently lost or are difficult to recognize (Setton and Sharma 2018). This may be the reason why the $b t d$-like gene escaped from recognition in an earlier analysis of gap and head gap gene-like genes in Euperipatoides (Janssen 2017a).

\section{Sp6-9 genes are conserved factors of appendage development in panarthropods}

In Euperipatoides, $S p 6-9$ is initially expressed in the brain that develops within the paired head lobes and in the most anterior trunk segments (Fig. S1). Within this domain, expression is stronger at the position where the appendages will form, but tissue between these limb primordia initially also expresses Sp6-9, albeit not so strongly (Fig. S1). The posterior segment addition zone (SAZ) and the last formed posterior segments do not express Sp6-9 (Fig. S1). Later, expression between the limb primordia disappears, resulting in a segmental pattern along the AP body axis in the regions where the limbs form (jaws, slime papillae and legs) (Fig. 2A). At subsequent developmental stages, this expression splits into a domain in the outgrowing limbs and expression in the tissue ventral to the base of the limbs that is likely contributing to the developing ventral nervous system (Fig. 2B, D, E).

In the growing appendages (Fig. 2B-F), expression is restricted to their distal ectoderm (Fig. 2G, H), while the proximal region does not express Sp6-9 (Fig. 2F). This overall expression pattern persists throughout development. !While the expression in the jaws, the slime papillae and the legs is restricted to the distal region, in the frontal appendages (the onychophoran antennae), the most anterior pair of appendages, the pattern is different. Here, Sp6-9 is expressed along the ventral ectoderm with exception of the very tip (Fig. 2AF). Expression of $S p 6-9$ in all appendages, except the frontal appendages, is virtually identical with the expression of Sp6-9 orthologs in other arthropods including Drosophila (Wimmer et al. 1996; Schaeper et al. 2009, 2010; Königsmann et al. 2017; Setton and Sharma 2018) and thus in line with a general role in limb growth.

The finding that the arthropod appendage-patterning key factor $\operatorname{Sp6-9}$ is expressed in conserved patterns in the onychophoran is not surprising given that also other conserved factors of arthropod appendage development such as Distalless (Dll), dachshund (dac) and homothorax (hth) are likely
Fig. 1 Phylogenetic analysis of Dmrt genes. Species abbreviations: Am, Apis mellifera (Hexapoda/Hymenoptera); Ek, Euperipatoides kanangrensis (Onychophora); Dm, Drosophila melanogaster (Hexapoda/ Diptera); Gm, Glomeris marginata (Myriapoda/ Diplopoda); $\mathrm{Ph}$, Parhyale hawaiensis (Pancrustacea/ Amphipoda); Pt, Parasteatoda tepidariorum (Chelicerata/ Aranea); Sm, Strigamia maritima (Myriapoda/Chilopoda). Green shade: Sp1-4 group. Blue shade: Sp6-9 group. Node support is given as posterior probabilities. Note that support for the Sp1-4 group and the Sp6-9 group is high, but Sp5/Btd-like factors do not form a clear monophyletic group

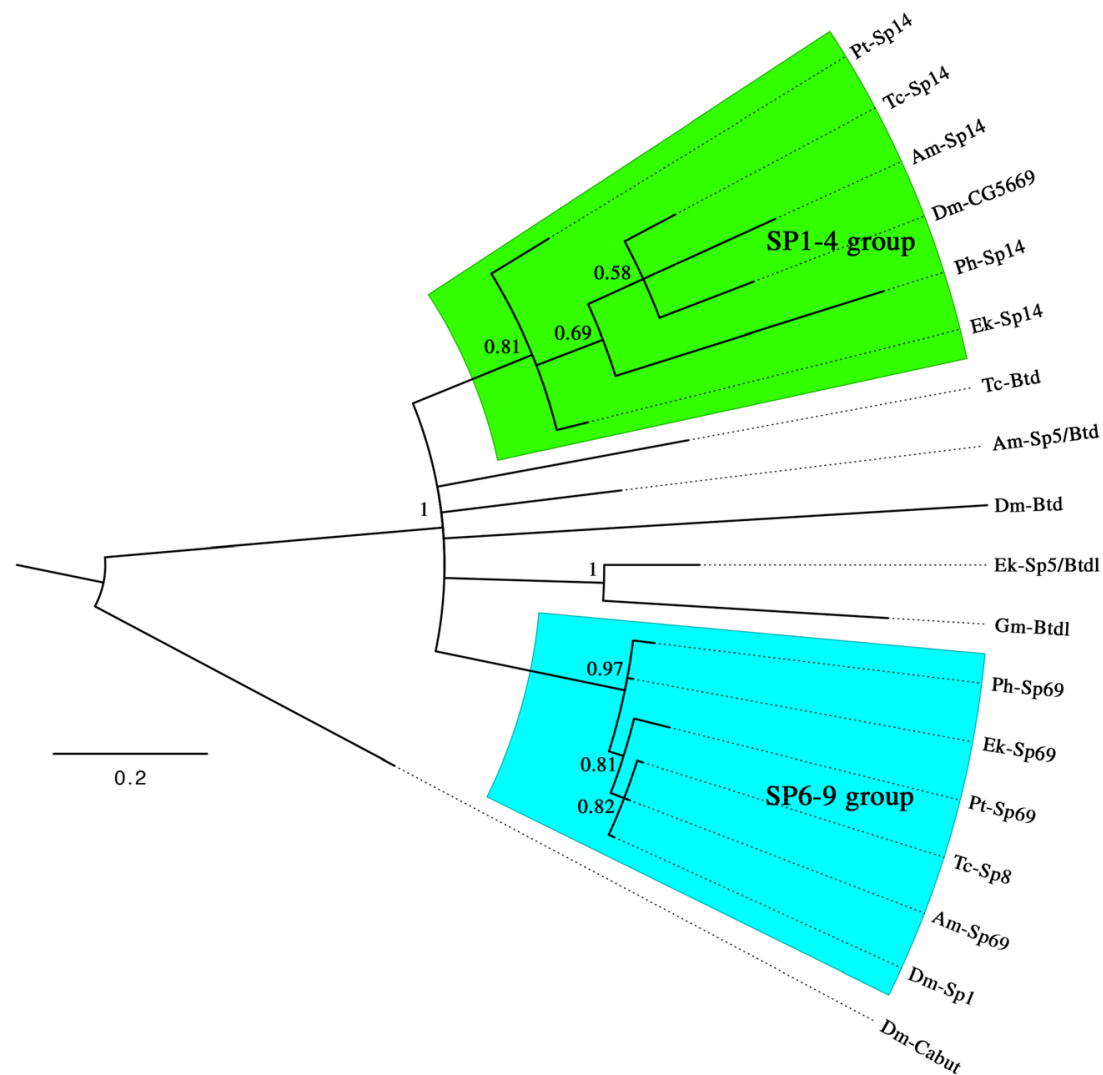




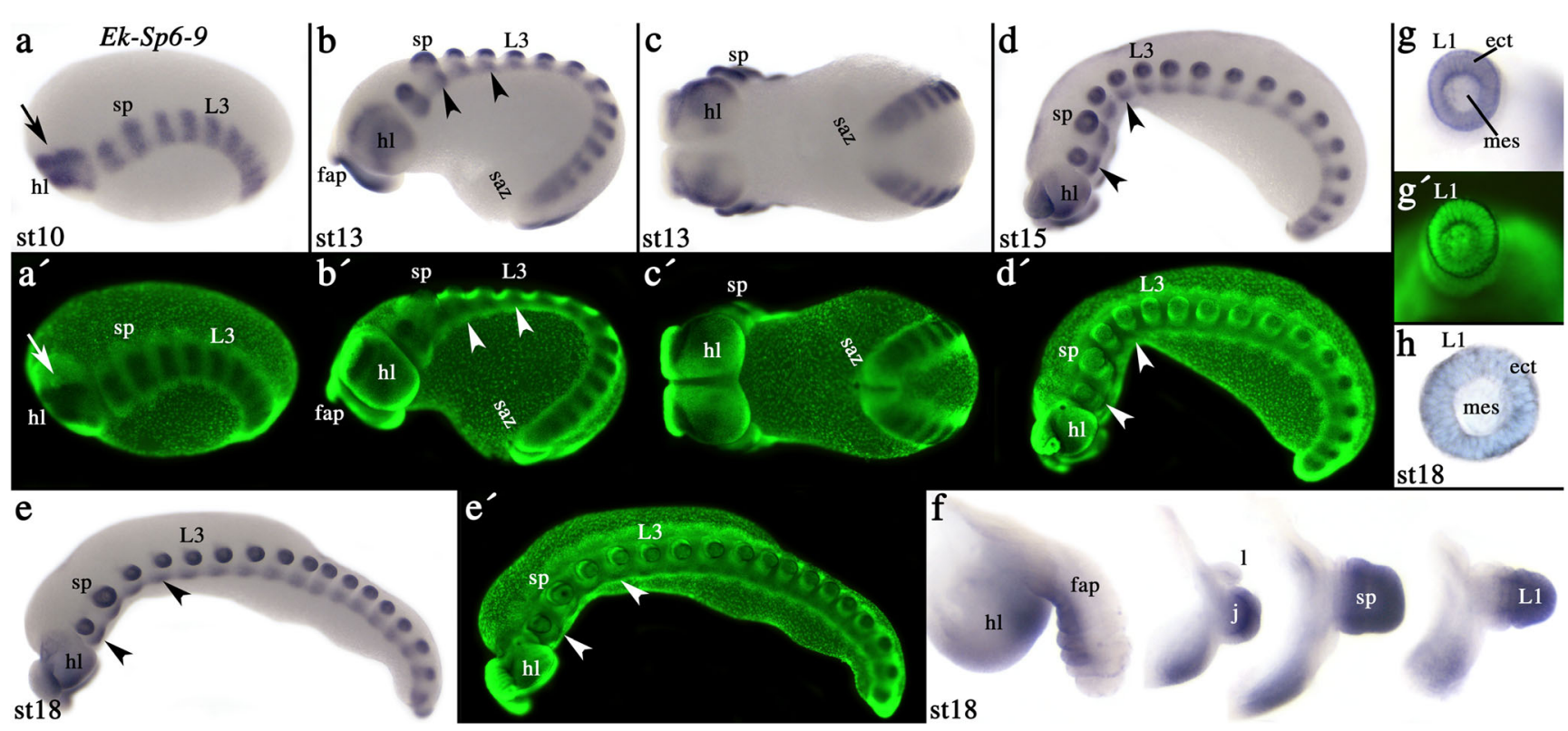

Fig. 2 Expression of Euperipatoides Sp6-9. In all panels, except panels (F) and $(\mathbf{G})$, anterior is to the left. Developmental stages are indicated. A Lateral view. The arrow points to the frontal appendage. B Lateral-ventral view. Arrowheads point to expression in the ventral nervous system. C Same embryo as in (B), ventral view. D and $\mathbf{E}$ Lateral views on later stage embryos. Arrowheads as in panel (C). F Dissected appendages. G On top view of a walking leg with removed tip. Anterior down. Note that

conserved in onychophorans (Angelini and Kaufman 2005; Janssen et al. 2010, 2015; Pechmann et al. 2010; Oliveira et al. 2014; Heingård et al. 2019). Altogether, these data indicate that the gene regulatory network orchestrating appendage growth is conserved in Panarthropoda.

The different expression of $\operatorname{Sp} 6-9$ in the frontal appendages (antennae; albeit not homologous with the antennae of Pancrustacea (e.g. Eriksson et al. 2010)) is remarkable. The frontal appendages are either considered to have evolved independently from the other appendages or represent highly derived serially homologous appendages, possibly homologous with the labrum of arthropods (reviewed in OrtegaHernández et al. 2017). If the former is true, expression patterns may be generally different, although conserved genetic networks may have been recruited for its development. If the latter is true, expression in the frontal appendages is best compared with the arthropod labrum, which is dorso-ventrally reversed due to rotation and fusion of these appendages in the lineage leading to Arthropoda (Kimm and Prpic 2006). Thus, genes that are expressed ventrally in the labrum are expressed dorsally in the other appendages and vice versa.

Despite the fact that $S p 6-9$ has been investigated in a wide range of arthropod species, expression in the labrum has unfortunately not been in the focus of these studies (Schaeper et al. 2009, 2010; Königsmann et al. 2017; Setton and Sharma 2018). In all hitherto investigated arthropod species, however, Sp6-9 is expressed in the labrum, and it appears that this expression is dorsal. We confirmed this by taking a closer look expression is in the ectoderm, but not the mesoderm. H On top view of a dissected walking leg with removed tip and base. The photograph shows a bright-field picture of a SYBR-Green counter-stained leg. $\mathbf{A}^{\prime}-$ $\mathbf{E}^{\prime}$ and $\mathbf{G}^{\prime}$ represent SYBR-Green counter-staining of the corresponding bright-field pictures. Abbreviations: ect, ectoderm; fap, frontal appendage; hl, head lobe; 1, lip; L, walking limb; mes, mesoderm; saz, segment addition zone; and sp, slime papilla

at the expression of $S p 6-9$ in the spider Parasteatoda tepidariorum (Fig. S2).

The dorsal expression of $\operatorname{Sp6-9}$ in the arthropod labrum and the ventral expression in the frontal appendage of onychophorans are thus conserved, supporting their possible homology. The overall gene expression profile and presence (or absence) of genes in the developing onychophoran frontal appendages and the labrum of arthropods, however, is not universally conserved (Janssen 2017b).

\section{Sp5/btd-like genes}

Embryonic expression of the onychophoran $S p 5 / b t d l$ gene is in some aspects similar to that of Sp6-9. Unlike Sp6-9, however, $S p 5 / b t d l$ is expressed very early during development in tissue around the blastopore (posterior pit) and the forming mouth-anus furrow (Fig. S3A, B), but note that the lips of the blastopore do not express Sp5/btdl (Fig. S3). When the furrow expands along the AP axis of the embryo, Sp5/btdl first remains expressed in its lips and in the tissue between the mouth-anus furrow and the embryo proper (Fig. S3C, D). Later, $S p 5 / b t d l$ disappears from this latter tissue and from the anterior of the mouth-anus furrow (the later mouth) (cf. Janssen et al. 2015)) (Fig. S3E); expression in the posterior of the furrow, the later anus, however, remains (Fig. S3E-G).

From stage 8 onwards, expression refines into transverse segmental stripes and a differentiated pattern in the posterior of the head lobes; the anterior of the head lobes remains free 
Fig. 3 Expression of Euperipatoides $\mathrm{Sp} 5 / \mathrm{btdl}$. In all panels, anterior is to the left. Developmental stages are indicated. A Lateral view. Note the segmental segmentation genelike pattern of $S p 5 / b t d l$. B Same embryo as in (A), ventral view. C Lateral view. The arrowhead points to expression in the ventral nervous system. D Dorsal view on anterior of embryo shown in (C). E Dorsal view of embryo shown in $(\mathbf{C})$. $\mathbf{F}$ and $\mathbf{G}$ Lateral views on later-stage embryos. Arrowheads as in panel $(\mathbf{C})$. $\mathbf{H}$ and I Ventral view on walking legs. Note expression in the tips of the legs. $\mathbf{A}^{\prime}$ and $\mathbf{E}^{\prime}$ represent SYBR-Green counter-staining of the corresponding bright-field pictures. Abbreviations as in Fig. 2, a, anus

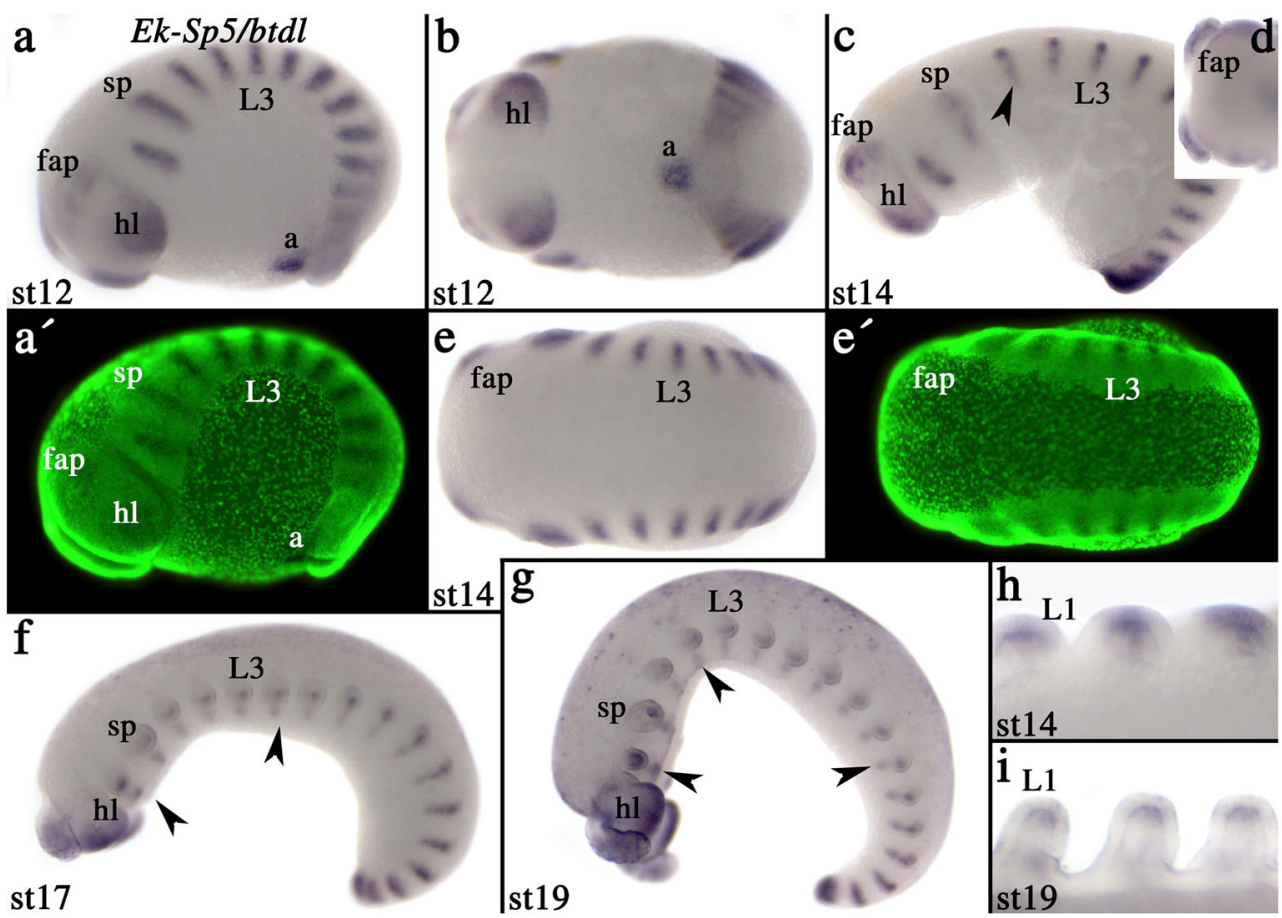

from expression (Fig. S3E-G and Fig. 3A, B). In contrast to Sp6-9, these segmental stripes are thinner. After limb growth begins, the stripes resolve into a pattern in the tips of the appendages and the ventral nervous system (much like for Sp6-9) (Fig. 3C, F, G). Expression in the appendages is restricted to the tips (Fig. 3D-I).

The expression profile of Euperipatoides Sp5/btdl is consistent with a role in the brain and ventral nervous system development as well as appendage development, each of which are characteristics of arthropod $S p 5 / b t d l$ genes (e.g. Estella et al. 2003; Schinko et al. 2008; Schaeper et al. 2010). Unlike in at least mandibulate arthropods where $S p 5 / b t d l$ is expressed early during development in a head gap gene-like domain (Wimmer et al. 1993; Schinko et al. 2008; Schaeper et al. 2010; Janssen et al. 2011; Hunnekuhl and Akam 2017; Jeon et al. 2019), and the gap gene function of $S p 6 / 9$ in a spider (Königsmann et al. 2017; Setton and Sharma 2018), there is no such pattern in the onychophoran that would suggest a similar function as a gap
Fig. 4 Expression of Euperipatoides Sp1-4. In all panels, anterior is to the left. Developmental stages are indicated. A Ventral view. B Lateral view. Note the segmentation-gene like expression of Sp1-4. C Ventral view. Note the strong expression in the posterior pit region. D Lateral view. Arrowheads point to expression in the ventral nervous system. E Close-up on the appendages of the embryo shown in (D). Ventral view. The arrow points to expression in the mesoderm. $\mathbf{A}^{\prime}-\mathbf{C}^{\prime}$ represent SYBRGreen counter-staining of the corresponding bright-field pictures. Abbreviations as in Fig. 2, a, anus; pp, posterior pit

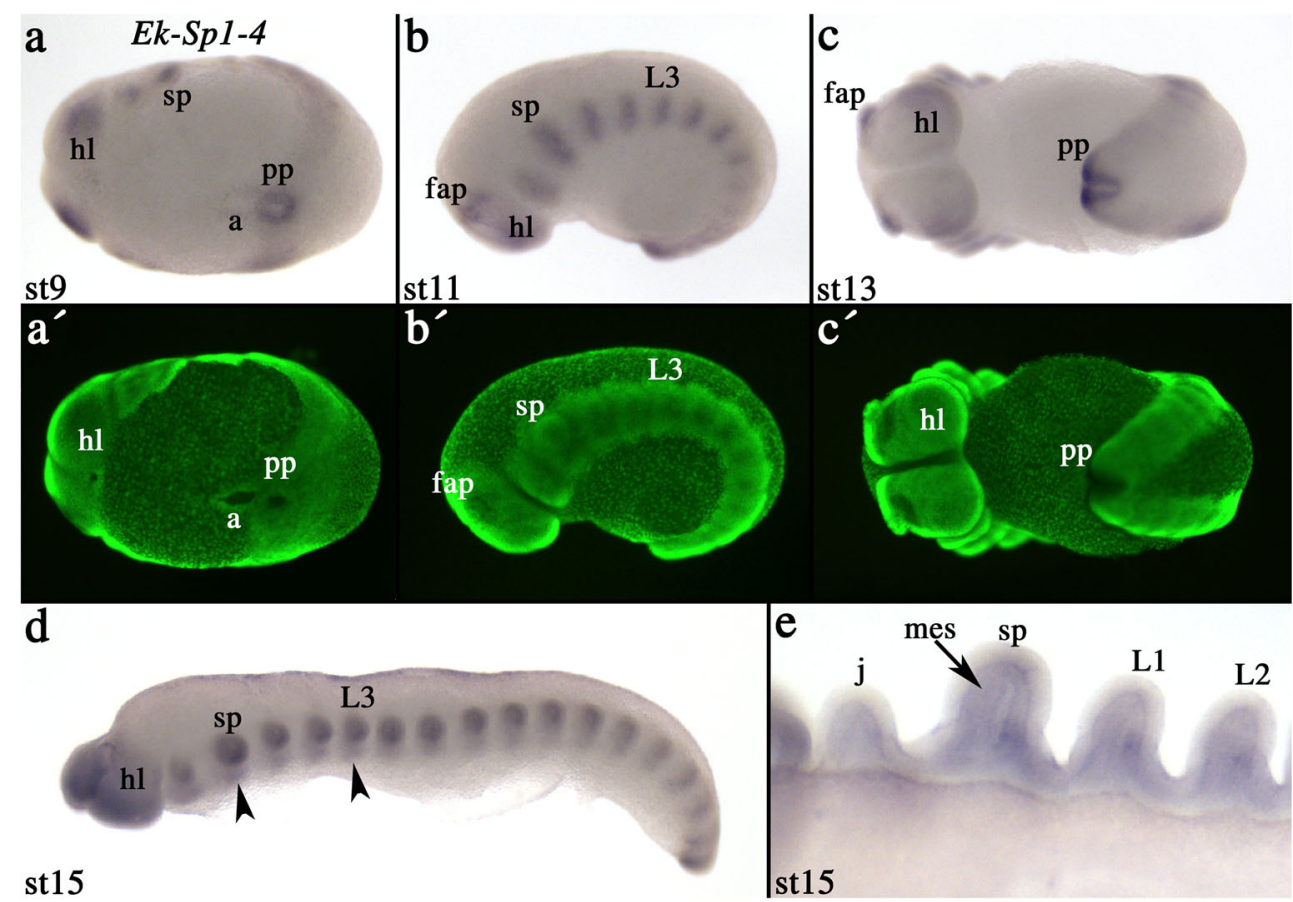


gene, neither of $S p 6 / 9$ nor of Sp5/btd (Supplementary Figs. S1 and S3). This may be little surprising given that the gap-gene-like network as known from Drosophila and as partially conserved in other arthropods (reviewed in, e.g. Damen 2007; Jaeger 2011) is not conserved in onychophorans (Franke and Mayer 2015; Janssen $2017 \mathrm{a}, \mathrm{b}$ ). What the early function of $S p 5 / b t d l$ in onychophorans is, however, remains unclear.

\section{Sp1-4 genes}

Data on arthropod Sp1-4 genes are restricted to the analysis of expression patterns. One reason for this may be the fact that hitherto investigated arthropod Sp1-4 genes are expressed ubiquitously during ontogenesis (summarized in Schaeper et al. 2010) and may thus represent universal transcription factors or may be regulated post-transcriptionally.

In the onychophoran, however, Sp1-4 is not expressed ubiquitously but rather in a pattern that is similar to that of the other two Sp genes (cf. Figs. 2, 3, and 4). Specifically, Euperipatoides Sp1-4 is expressed in the brain, but not in exactly the same pattern as either $S p 5 / b t d l$ or $S p 6-9$ (Fig. 4A-C), and as for $S p 5 / b t d l$ and $S p 6-9$, in the form of transverse segmental stripes that later during development split into a domain in the limbs and a domain in the developing ventral nervous system (Fig. 4D/E). Unlike $S p 5 / b t d l$ and $S p 6-$ 9, Spl-4 is also expressed in the posterior pit that may suggest a function in segment addition and/or posterior elongation (Fig. 4A/C). Expression in the developing appendages is mainly restricted to the mesoderm (Fig. 4E).

Authors' contributions All experimental work was performed by RJ. The first draft of the manuscript was written by RJ. Both authors wrote the final version of this manuscript.

Funding information Open access funding provided by Uppsala University. Financial funding was provided by the Swedish Natural Science Council (VR) (grant no. 621-2011-4703) and the Marie Skłodowska-Curie Action (MSCA), Innovative Training Network (ITN), H20202-MSCA-ITN-2017 "EvoCELL" (grant no. 766053). We are thankful for the support of the New South Wales Government Department of Environment and Climate Change by provision of a permit SL100159 to collect onychophorans at Kanangra-Boyd National Park.

Open Access This article is licensed under a Creative Commons Attribution 4.0 International License, which permits use, sharing, adaptation, distribution and reproduction in any medium or format, as long as you give appropriate credit to the original author(s) and the source, provide a link to the Creative Commons licence, and indicate if changes were made. The images or other third party material in this article are included in the article's Creative Commons licence, unless indicated otherwise in a credit line to the material. If material is not included in the article's Creative Commons licence and your intended use is not permitted by statutory regulation or exceeds the permitted use, you will need to obtain permission directly from the copyright holder. To view a copy of this licence, visit http://creativecommons.org/licenses/by/4.0/.

\section{References}

Angelini DR, Kaufman TC (2005) Insect appendages and comparative ontogenetics. Dev Biol 286:57-77

Beermann A, Aranda M, Schröder R (2004) The Sp8 zinc-finger transcription factor is involved in allometric growth of the limbs in the beetle Tribolium castaneum. Development. 131:733-742

Campbell LI, Rota-Stabelli O, Edgecombe GD, Marchioro T, Longhorn SJ, Telford MJ, Philippe H, Rebecchi L, Peterson KJ, Pisani D (2011) MicroRNAs and phylogenomics resolve the relationships of Tardigrada and suggest that velvet worms are the sister group of Arthropoda. Proc Natl Acad Sci U S A 108:15920-15924

Cordoba S, Requena D, Jory A, Saiz A, Estella C (2016) The evolutionarily conserved transcription factor $\mathrm{Sp} 1$ controls appendage growth through notch signaling. Development. 143:3623-3631

Damen WG (2007) Evolutionary conservation and divergence of the segmentation process in arthropods. Dev Dyn 236:1379-1391

Dynan WS, Tjian R (1983) Isolation of transcription factors that discriminate between different promoters recognized by RNA polymerase II. Cell 32:669-680

Eriksson BJ, Tait NN, Budd GE, Janssen R, Akam M (2010) Head patterning and Hox gene expression in an onychophoran and its implications for the arthropod head problem. Dev Genes Evol 220:117122

Estella C, Mann RS (2010) Non-redundant selector and growthpromoting functions of two sister genes, buttonhead and $S p 1$, in Drosophila leg development. PLoS Genet 6:e1001001

Estella C, Rieckhof G, Calleja M, Morata G (2003) The role of buttonhead and $\mathrm{Sp} 1$ in the development of the ventral imaginal discs of Drosophila. Development 130:5929-5941

Franke FA, Mayer G (2015) Expression study of the hunchback ortholog in embryos of the onychophoran Euperipatoides rowelli. Dev Genes Evol 225:207-219

Giribet G, Edgecombe GD (2017) Current understanding of Ecdysozoa and its internal phylogenetic relationships. Integr Comp Biol 57: 455-466

Gouy M, Guindon S, Gascuel O (2010) SeaView version 4: a multiplatform graphical user interface for sequence alignment and phylogenetic tree building. Mol Biol Evol 27:221-224

Heingård M, Turetzek N, Prpic NM, Janssen R (2019) FoxB, a new and highly conserved key factor in arthropod dorsal-ventral (DV) limb patterning. Evodevo 10:28. https://doi.org/10.1186/s13227-0190141-6

Hogvall M, Schönauer A, Budd GE, McGregor AP, Posnien N, Janssen $\mathrm{R}$ (2014) Analysis of the Wnt gene repertoire in an onychophoran provides new insights into the evolution of segmentation. Evodevo 5:14

Huelsenbeck JP, Ronquist F (2001) MRBAYES: bayesian inference of phylogenetic trees. Bioinformatics 17:754-755

Hunnekuhl VS, Akam M (2017) Formation and subdivision of the head field in the centipede Strigamia maritima, as revealed by the expression of head gap gene orthologues and hedgehog dynamics. Evodevo 8:18

Jaeger J (2011) The gap gene network. Cell Mol Life Sci 68:243-274

Janssen R (2017a) A molecular view of onychophoran segmentation. Arthropod Struct Dev 46:341-353

Janssen R (2017b) Comparative analysis of gene expression patterns in the arthropod labrum and the onychophoran frontal appendages, and its implications for the arthropod head problem. Evodevo 8(1). https://doi.org/10.1186/s13227-016-0064-4

Janssen R, Budd GE (2013) Deciphering the onychophoran 'segmentation gene cascade': gene expression reveals limited involvement of pair rule gene orthologs in segmentation, but a highly conserved segment polarity gene network. Dev Biol 382:224-234 
Janssen R, Eriksson BJ, Budd GE, Akam M, Prpic NM (2010) Gene expression patterns in an onychophoran reveal that regionalization predates limb segmentation in pan-arthropods. Evol Dev 12:363372

Janssen R, Budd GE, Damen WG (2011) Gene expression suggests conserved mechanisms patterning the heads of insects and myriapods. Dev Biol 357:64-72

Janssen R, Jörgensen M, Lagebro L, Budd GE (2015) Fate and nature of the onychophoran mouth-anus furrow and its contribution to the blastopore. Proc Biol Sci 282:1805

Janssen R, Andersson E, Betnér E, Bijl S, Fowler W, Höök L, Leyhr J, Mannelqvist A, Panara V, Smith K, Tiemann S (2018) Embryonic expression patterns and phylogenetic analysis of panarthropod sox genes: insight into nervous system development, segmentation and gonadogenesis. BMC Evol Biol 18:88

Jeon H, O J, Jin S, Lim J, Choe CP (2019) A role for buttonhead in the early head and trunk development in the beetle Tribolium castaneum. Dev Reprod 23:63-72

Kadonaga JT, Carner KR, Masiarz FR, Tjian R (1987) Isolation of cDNA encoding transcription factor $\mathrm{Sp} 1$ and functional analysis of the DNA binding domain. Cell 51:1079-1090

Kimm MA, Prpic NM (2006) Formation of the arthropod labrum by fusion of paired and rotated limb-bud like primordial. Zoomorphology 125:147e155

Kolell KJ, Crawford DL (2002) Evolution of Sp transcription factors. Mol Biol Evol 19:216-222

Königsmann T, Turetzek N, Pechmann M, Prpic NM (2017) Expression and function of the zinc finger transcription factor $S p 6-9$ in the spider Parasteatoda tepidariorum. Dev Genes Evol 227:389-400

Notredame C, Higgins DG, Heringa J (2000) T-Coffee: a novel method for fast and accurate multiple sequence alignment. J Mol Biol 302: 205-217

Oliveira MB, Liedholm SE, Lopez JE, Lochte AA, Pazio M, Martin JP, Mörch PR, Salakka S, York J, Yoshimoto A, Janssen R (2014) Expression of arthropod distal limb-patterning genes in the onychophoran Euperipatoides kanangrensis. Dev Genes Evol 224:8796

Ortega-Hernández J, Janssen R, Budd GE (2017) Origin and evolution of the panarthropod head - a palaeobiological and developmental perspective. Arthropod Struct Dev 46:354-379
Pechmann M, Khadjeh S, Sprenger F, Prpic NM (2010) Patterning mechanisms and morphological diversity of spider appendages and their importance for spider evolution. Arthropod Struct Dev 39:453-467

Schaeper ND, Prpic NM, Wimmer EA (2009) A conserved function of the zinc finger transcription factor $S p 8 / 9$ in allometric appendage growth in the milkweed bug Oncopeltus fasciatus. Dev Genes Evol 219:427-435

Schaeper ND, Prpic NM, Wimmer EA (2010) A clustered set of three Spfamily genes is ancestral in the Metazoa: evidence from sequence analysis, protein domain structure, developmental expression patterns and chromosomal location. BMC Evol Biol 30(10):88

Schinko JB, Kreuzer N, Offen N, Posnien N, Wimmer EA, Bucher G (2008) Divergent functions of orthodenticle, empty spiracles and buttonhead in early head patterning of the beetle Tribolium castaneum (Coleoptera). Dev Biol 317:600-613

Schöck F, Purnell BA, Wimmer EA, Jäckle H (1999) Common and diverged functions of the Drosophila gene pair $D-S p 1$ and buttonhead. Mech Dev 89:125-132

Setton EVW, Sharma PP (2018) Cooption of an appendage-patterning gene cassette in the head segmentation of arachnids. Proc Natl Acad Sci U S A 115:E3491-E3500

Suske G (1999) The Sp-family of transcription factors. Gene 238:291300

Suske G (2017) NF-Y and SP transcription factors - new insights in a long-standing liaison. Biochim Biophys Acta Gene Regul Mech 1860:590-597

Suske G, Bruford E, Philipsen S (2005) Mammalian SP/KLF transcription factors: bring in the family. Genomics 85:551-556

Wimmer EA, Jäckle H, Pfeifle C, Cohen SM (1993) A Drosophila homologue of human $\mathrm{Sp} 1$ is a head-specific segmentation gene. Nature 366:690-694

Wimmer EA, Frommer G, Purnell BA, Jäckle H (1996) Buttonhead and D-Sp1: a novel Drosophila gene pair. Mech Dev 59:53-62

Zhao C, Meng A (2005) Sp1-like transcription factors are regulators of embryonic development in vertebrates. Develop Growth Differ 47: 201-211

Publisher's note Springer Nature remains neutral with regard to jurisdictional claims in published maps and institutional affiliations. 